\title{
Hematological Profile and Gametocyte Carriage in Malaria Patients from Southern Pakistan
}

Najia K. Ghanchi ${ }^{1}$, Mohammad Hassaan Khan ${ }^{2}$, Muhammad Abdullah Arain ${ }^{2}$, Mustafa Bin Ali Zubairi ${ }^{3}$, Ahmed Raheem ${ }^{1}$, Muhammad A. Khan ${ }^{4}$, Mohammad A. Beg ${ }^{1}$

1. Pathology, Aga Khan University Hospital, Karachi, PAK 2. Pathology and Laboratory Medicine, Aga Khan University Hospital, Karachi, PAK 3. Pathology and Laboratory Medicine, Dow University of Health Sciences (DUHS), Karachi, PAK 4. Community Health Sciences, Aga Khan University Hospital, Karachi, PAK

Corresponding author: Mohammad A. Beg, masim.beg@aku.edu

\section{Abstract \\ Background}

Malarial infection is a major cause of concern, both worldwide and in Pakistan. Gametocytes are the sexual forms of the parasite that are essential for transmission. They fuse inside the mosquito to develop sporozoites. Gametocytes of the plasmodium parasites, which cause the infection, differentiate into male and female gametocytes. These gametocytes constitute the sexual stage of the malaria parasite and are essential in transmission of the disease from human to vector Anopheles. Gametocytes are affected by factors such as host immunity, drug treatment, reticulocytemia, anemia, low levels of asexual parasitemia and stress to the parasite. The aim of this study was to observe the hematological parameters, age and gametocyte carriage in an area of seasonal malaria transmission.

\section{Methods}

The study was conducted at Aga Khan University Hospital (AKUH) Laboratory over the period of one year and 294 patients with uncomplicated malaria were recruited. Patients infected with Plasmodium falciparum (P. falciparum) or Plasmodium vivax (P. vivax) malaria and no co-morbidities were included in the study.

\section{Results}

Gametocytemia was highest during the period of July to November, with P. vivax, 267 (90.8\%), predominating compared to P. falciparum, 27 (9.2\%). P. vivax gametocytes were observed from May to October and P. falciparum gametocytes were observed from July to December. Low hemoglobin in females and low platelet levels were observed. The mean platelet count was significantly lower in cases of P. vivax having gametocytes compared to P. falciparum with gametocytes. Higher parasitic index was associated with lower platelet count. The most significantly altered parameters were hemoglobin, hematocrit, white blood cell (WBC), and platelet count. Hemoglobin and platelets were significantly lower during the malaria season in study participants, both male and female.

Received 02/08/2019

Review began 02/22/2019 Review ended 03/05/2019 Published 03/15/2019

\section{() Copyright 2019}

Ghanchi et al. This is an open access article distributed under the terms of the Creative Commons Attribution License CC-BY 3.0., which permits unrestricted use, distribution, and reproduction in any medium, provided the original author and source are credited.

\section{Conclusion}

In conclusion, infection with P. falciparum and P. vivax modulates significant changes in hematological parameters in populations living in malaria endemic regions. In the malaria season males were more frequently affected by malaria with thrombocytopenia. Gametocyte carriage remains unaffected by seasonal changes thus ensuring parasite transmission during the dry season.

Categories: Pathology, Infectious Disease, Other

Keywords: gametocytes, plasmodium falciparum, plasmodium vivax, temperature

\section{Introduction}

Malaria is one of the leading causes of morbidity and mortality worldwide, with nearly half of the world population classified as 'at risk' in 2015. According to the World Health Organization (WHO), there were around 212 million malaria cases reported and an estimated 490,000 deaths caused by malaria in 2015 worldwide [1]. Pakistan is one of the countries affected, with 177 million out of the 185 million population being at risk, along with 0.9 million presumed and confirmed cases every year. Malaria transmission is seasonal and coincides with the monsoon season. Plasmodium falciparum (P. falciparum) and Plasmodium vivax (P. vivax) coexist in Pakistan and transmission is highest from July to November. Most of the Plasmodia after infecting the host undergo asexual replication, but a proportion differentiates into gametocytes. Gametocytes constitute the sexual stages of the malaria parasite and are essential in the transmission of the disease from human to its vector female Anopheles mosquito [2].

Gametocytes of P. falciparum are sequestered away from the peripheral circulation for maturation, and 
evidence has been presented for the development of gametocytes in the hematopoietic system of the human bone marrow [3]. It has also been postulated that the bone marrow could be a niche for gametocyte production and maturation and/or a reservoir in P. vivax infections. Thus, gametocytes appear in the blood stream seven to 15 days after, for P. falciparum, but much sooner, for P. vivax, after the initial appearance of asexual stage parasites [4].

While there is much information about the asexual forms of the parasite, not much is known of the sexual forms and the process of gametocytogenesis. Knowledge of factors inducing or affecting gametocytogenesis/gametogenesis may help facilitate actions which could disrupt the malaria transmission cycle. The conversion of asexual to sexual stages depends upon factors such as host immunity, drug treatment, presence of reticulocytes (in vitro), anemia, low levels of asexual parasitemia and stress to the parasite [5]. However, the stress may not be essential for gametocytogenesis, shown by the commitment to sexual development at early infection stages [6].

Presence of anemia is identified as a risk factor for gametocytaemia [7]. Factors that decrease the survival of asexual forms of the parasite, can lead to an increase in density of sexual forms. Anemia may lead to a greater chance of gametocyte formation [8]. Anemia may also lead to increased reticulocyte levels in the blood and reticulocyte-rich blood has been found to promote enhanced gametocyte formation in vitro [9-10].

Host immunity also plays a significant part in gametocyte production. Gametocytogenesis was induced in vitro for P. falciparum using lymphocytes and serum, acquired from P. falciparum-infected children [11]. Therefore, the host immunity not only increased gametocyte production directly, but also indirectly, by killing asexual stages [12].

Gametocyte carriage has also been shown to differ in different age groups and seasons. Gametocytemia is reported to be greater in the younger age groups as compared to the older, especially those aged $<16$ years [13]. Increased gametocyte carriage was observed during the dry season in patients residing on the ThaiBurmese border [14]. Furthermore, the continuous use of chloroquine (CQ) and sulphadoxinepyrimethamine monotherapy in resistant infections results in increased gametocytemia and hence, increased transmission [15-16].

This study aims to document and compare the changes in hematological parameters of the malaria-infected patients, along with the variations in gametocyte carriage and malaria transmission during different seasons. This can help in a better understanding of malaria transmission and prognosis and according to a study conducted, these hematological variations when used in combination with other clinical and microscopy methods can improve malaria diagnosis and treatment [17].

In this study the changes in the hematological parameters, age and seasonal variation of gametocyte carriage were observed in an endemic area.

\section{Materials And Methods}

The study was conducted at Aga Khan University Hospital (AKUH) Laboratory, Karachi, Pakistan. A total of 294 patients infected with P. falciparum or P. vivax malaria were included. Patients who were malaria negative and refused to consent were excluded.

The study was approved by the Ethical Review Committee of Aga Khan University Hospital and conducted in accordance with the Good Clinical Practice of Declaration of Helsinki. Informed consent was obtained from enrolled patients.

Peripheral blood $(1 \mathrm{ml})$ was obtained from the participants for detection of the malaria parasite. Thick and thin smears were prepared from each patient's blood, and Giemsa staining performed, to quantify and identify the species of the parasite respectively. The malaria positive blood films were reviewed by an expert. Parasitemia was calculated using 100x oil immersion microscopy. The parasites were counted against 200 white blood cells (WBCs) and the parasite density was calculated with the help of the total leukocyte count (TLC). Participants were stratified into three groups based on age less than 15 years, 16-40 years and greater than 40 years.

Data was analyzed using SPSS Statistics for Windows, Version 21.0 (IBM Corp., Armonk, NY, USA). Arithmetical means and medians were calculated, where applicable, for all continuous baseline variables. Mann-Whitney U test and ANOVA test, if the assumption was found, then Kruskal-Wallis test were used to compare differences between different groups. Multivariate binary logistic regression was applied to compare the cause and effect among hematological parameters and demographics variable. p-value $<=0.05$ was taken as significance at two-sided p-value.

\section{Results}

In this study of 294 malaria-infected patients, 207 were males (70.4\%) and 87 were females (29.6\%). 


\section{Cureus}

Gametocytemia with P. vivax, 267 (90.8\%), was observed to predominate compared to P. falciparum, 27 (9.2\%). P. vivax gametocytes were observed from May to October and P. falciparum gametocytes were noted from July to December.

The mean hemoglobin $(\mathrm{Hb})$ and hematocrit (HCT) levels in females were observed to be low whereas, for males they were in the low-normal range (Table 1).

\begin{tabular}{|c|c|c|c|}
\hline \multirow{2}{*}{ Blood Parameters } & Male $(n=207)$ & Female $(n=87)$ & \multirow{2}{*}{ p-value } \\
\hline & Mean \pm SD & Mean \pm SD & \\
\hline Hemoglobin (g/dl) & $11.85 \pm 1.09$ & $10.61 \pm 0.58$ & $<0.0001^{*}$ \\
\hline Hematocrit (\%) & $35.06 \pm 3.21$ & $31.84 \pm 1.86$ & $<0.0001^{*}$ \\
\hline Red blood cell count (x1012L) & $4.26 \pm 0.11$ & $3.90 \pm 0.30$ & $<0.0001^{*}$ \\
\hline White blood cell count ( $x 109 L)$ & $6.53 \pm 0.82$ & $5.71 \pm 0.31$ & $<0.0001^{*}$ \\
\hline Platelets (x109L) & $89.94 \pm 5.64$ & $103.9 \pm 10.50$ & $<0.0001^{\star}$ \\
\hline
\end{tabular}

TABLE 1: Hematological parameters of patients infected with Plasmodium with respect to gender.

* Normal values - Hemoglobin: 11.1-14.5 g/dl, hematocrit: $35.4-42 \%$, total leukocyte count: 4-10 x $10^{9} \mathrm{~L}$, platelets: $150-400 \times 10^{9} \mathrm{~L}$, red blood cell: $3.9-5.5 \times 10^{12} \mathrm{~L}$

On comparison of the different age groups, the $\mathrm{Hb}$ and HCT levels of the patients less than 15 years were found to be the most significantly affected (Table 2). A normal WBC count, along with thrombocytopenia, was seen in both male and female patients of all age groups. Excluding WBC count, all the other hematological parameters were significantly different between males and females $(p<0.0001)$.

\begin{tabular}{|c|c|c|c|c|c|}
\hline Hematological Parameters & $<=15$ Years $(n=36)$ & $16-40$ Years $(n=145)$ & $>40$ Years $(n=113)$ & Total $(n=294)$ & p-value \\
\hline $\mathrm{Hb}$ & $10.28 \pm 2.15$ & $12.41 \pm 2.18$ & $11.71 \pm 2.34$ & $11.86 \pm 2.34$ & $<0.001^{*}$ \\
\hline White blood cell (WBC) & $5.82 \pm 3.07$ & $5.85 \pm 2.37$ & $6.92 \pm 3.97$ & $6.26 \pm 3.2$ & $0.018^{\star}$ \\
\hline НСТ & $30.65 \pm 6.02$ & $36.77 \pm 6.3$ & $34.77 \pm 6.93$ & $35.19 \pm 6.8$ & $<0.001^{*}$ \\
\hline Platelets & 96.5 (114.5-49.5) & $82.5(120.2-53)$ & $86(127-48.5)$ & $85(120-50.5)$ & 0.933 \\
\hline RBC & $3.98 \pm 0.8$ & $4.34 \pm 0.75$ & $4.06 \pm 0.85$ & $4.19 \pm 0.81$ & $0.005^{\star}$ \\
\hline
\end{tabular}

TABLE 2: Hematological parameters of patients infected with Plasmodium with respect to different age groups.

* Normal values - Hemoglobin (Hb): $11.1-14.5 \mathrm{~g} / \mathrm{dl}$, hematocrit (HCT): $35.4-42 \%$, total leukocyte count: $4-10 \times 10^{9} \mathrm{~L}$, platelets: $150-400 \times 10^{9} \mathrm{~L}$, red blood cell (RBC): $3.9-5.5 \times 10^{12} \mathrm{~L}$

There was a significant difference in all hematological parameters between $\mathrm{P}$. falciparum and P. vivax, except for the platelet levels. The HCT $(p=0.000), \mathrm{Hb}(\mathrm{p}=0.000)$ and red blood cell $(\mathrm{RBC})(\mathrm{p}=0.000)$ levels were all higher in P. vivax-infected patients while the WBC $(p=0.000)$ and platelet $(p=0.090)$ levels were greater in those suffering from P. falciparum infection (Table 3). Binary logistic regression analysis was performed between gametocytemia and independent variables such as RBC, platelet and $\mathrm{Hb}$. RBC count were significantly lower with higher gametocytemia odd ratio $0.467(0.251-0.867, \mathrm{P}=0.016 *)$, male gender were more significantly 12.6 times gametocytaemia higher in Plasmodium vivax as compared to female subjects ( $\mathrm{p}$-value $<0.001^{*}$ ) while other parameters were not significantly associated with gametocytemia. Univariate analysis revealed that gametocyte carriage decreased with age, the proportion of gametocyte positive patients was highest in the younger age groups and decreased rapidly with age. 


\section{Cureus}

\begin{tabular}{|c|c|c|c|}
\hline \multirow{2}{*}{ Hematological Parameters } & P. falciparum $(n=27)$ & P. vivax $(n=267)$ & p-value* \\
\hline & Mean \pm SD & Mean \pm SD & \\
\hline Hemoglobin (g/dl) & $8.54 \pm 1.25$ & $12.19 \pm 2.15$ & $<0.001$ \\
\hline Hematocrit (\%) & $24.49 \pm 3.6$ & $36.22 \pm 6.16$ & $<0.001$ \\
\hline Red blood cell count (x1012L) & $2.90 \pm 0.42$ & $4.32 \pm 0.72$ & $<0.001$ \\
\hline White blood cell count (x109L) & $9.52 \pm 5.7$ & $5.93 \pm 2.6$ & $<0.001$ \\
\hline Platelets (x109L) & $117.89 \pm 138$ & $94.09 \pm 58$ & $0.09^{\star}$ \\
\hline
\end{tabular}

\section{TABLE 3: Hematological parameter of patients infected with P. falciparum and P. vivax.}

* Normal values - Hemoglobin: 11.1-14.5 g/dl, hematocrit: 35.4-42\%, total leukocyte count: 4-10 x $10^{9} \mathrm{~L}$, platelets: $150-400 \times 10^{9} \mathrm{~L}$; red blood cell: $3.9-5.5 \times 10^{12} \mathrm{~L}$

Gametocyte density for P. vivax peaked earlier, around July-August, while that of P. falciparum came later, around September-October. On the other hand, the mean Hb, HCT, RBC and WBC levels were found to be similar in both the rainy and dry seasons but, the mean platelet levels were lower in the rainy season as compared to the dry season (Table 4).

\begin{tabular}{|c|c|c|c|}
\hline \multirow{2}{*}{ Blood Parameters } & Rainy season $(n=49)$ & Dry season $(n=245)$ & \multirow{2}{*}{ p-value* } \\
\hline & Mean \pm SD & Mean \pm SD & \\
\hline Hemoglobin (g/dl) & $8.54 \pm 1.25$ & $12.19 \pm 2.15$ & 0.8096 \\
\hline Hematocrit (\%) & $24.49 \pm 3.6$ & $36.22 \pm 6.16$ & 0.9918 \\
\hline Red blood cell count ( $x 1012 \mathrm{~L}$ ) & $4.22 \pm 0.82$ & $4.06 \pm 0.77$ & 0.1655 \\
\hline White blood cell count (x109 L) & $6.14 \pm 2.87$ & $6.70 \pm 4.20$ & 0.2191 \\
\hline Platelets (x109 L) & $94.10 \pm 68.62$ & $104.24 \pm 72.26$ & 0.3049 \\
\hline
\end{tabular}

\section{TABLE 4: Effects of seasonal variation in hematological parameter of patients with} gametocytaemia.

* Normal values - Hemoglobin: 11.1-14.5 g/dl, hematocrit: $35.4-42 \%$, total leukocyte count: $4-10 \times 10^{9} \mathrm{~L}$, platelets: $150-400 \times 10^{9} \mathrm{~L}$, red blood cell: $3.9-5.5 \times 10^{12} \mathrm{~L}$

Bar charts represent gametocyte carriage of both P. falciparum and P. vivax detected in over a year period. Percent gametocytemia detected monthly are indicated. Average monthly temperatures recorded are plotted and indicated as red line. High gametocytes carriage was noted during malaria season from June to October when temperatures were higher (above $30^{\circ} \mathrm{C}$ ). Gametocytemia was seen to increase as the temperature increased and was high from May to November as shown in Figure 1. 


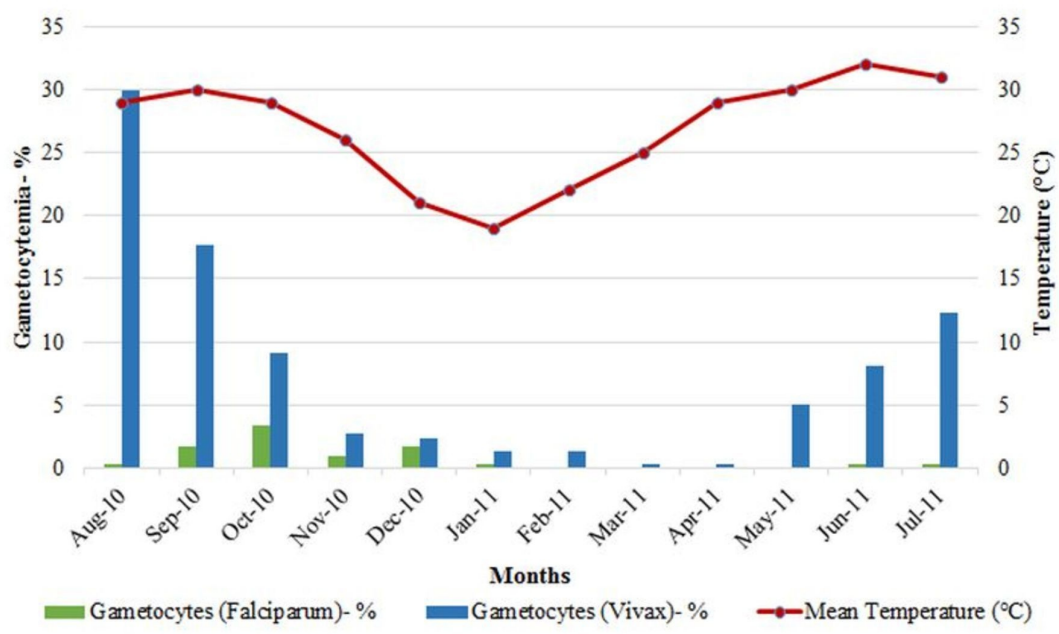

FIGURE 1: Monthly variation in gametocytes carriage and its association with rising temperatures.

\section{Discussion}

The two most common malaria species, Plasmodium falciparum (P. falciparum) and Plasmodium vivax (P. vivax), have overlapping areas of distribution in Pakistan. Even though there is a higher incidence of $\mathrm{P}$. falciparum infections throughout the world, our study showed a higher incidence of P. vivax species ( $90.8 \%$ ) compared to P. falciparum (9.2\%). These findings are consistent with the infecting patterns of this region [18-19]. We have also reconfirmed that gametocyte carriage was 4.8 times more common in vivax vs. falciparum patients [6]. Presence of gametocytes (P. vivax and P. falciparum) was seen in patients and analyzed according to their age. Univariate analysis revealed gametocyte carriage decreased with age, the proportion of gametocyte positive patients was highest in the younger age groups and decreased rapidly with age.

In this study, the changes in hematological parameters of the malaria-infected patients were observed. The platelet counts were found to be significantly low in all age groups, for both genders. On the other hand, the female patients were diagnosed with anemia. Anemia was common in female patients compared to male ( $\mathrm{p}$ value $<0.001 *$ ).

The majority of the Pakistani population suffers from anemia, especially iron deficiency anemia, and all age groups, from infants to elderly, are affected [20]. Anemia can be attributed to the destruction of red blood cells, splenic removal of parasitized and non-parasitized RBCs and dyserythropoiesis and engulfment in reticulo-endothelial system [21]. Anemia due to malaria is indicated by the destruction of both infected and uninfected RBCs, a reduction in erythrocyte precursors and inhibition of erythropoiesis eventually leading to severe malaria or death in patients. This is commonly seen in infections by P. falciparum species. Hemozoin is believed to play a major part in this by having an influence on the extrinsic apoptotic pathway of erythroid cells [22]. On the other hand, thrombocytopenia is a common laboratory observation in malaria patients and the exact etiology is not very well understood. The popular postulations suggest that macrophage-mediated immunologic lysis, splenic sequestration, ultra-structural changes and dyspoietic development in the bone marrow could be the likely causes [23]. Severe disease could also lead to disseminated intravascular coagulation (DIC) which would cause increased consumption of platelets causing thrombocytopenia in the process.

There have been different observations regarding the changes in WBC count due to malaria. A study conducted in Kenya stated that malaria causes leukocytosis, and this was associated with a worse prognosis [24]. Whereas, in another study near the Thailand-Myanmar border, the WBC count in the malaria-infected patients was seen to increase [25]. In our study, the WBC count of the patients was mostly in the normal range. As a result, we advise that the possibility of a normal WBC count in a malaria-infected patient along with thrombocytopenia and anemia should always be taken into consideration.

Malaria incidence usually has a seasonal repetition because the life span, population and rate of survival of the Anopheles mosquito depend upon climatological elements. Pakistan is a mesoendemic region which means there is a regular seasonal transmission which occurs under normal rainfall conditions [26]. In this study, the highest rate of gametocytemia was seen during the rainy season in the second half of the year. This time period lies in the high transmission season for malaria infectivity for Pakistan and corresponds to 
high temperatures and monsoon rainfall in the region, both factors that facilitate infection transmission. However, during the dry season a reservoir of gametocytes persists. P. falciparum gametocytes may persist for three to six weeks after the cure of infection [27] and analysis of a study revealed that P. vivax gametocyte appeared for up to 63 days after enrollment for treatment [28]. Hence, cases of gametocyte carriage have been reported from December to February facilitating malaria transmission. Gametocytaemia persists throughout the dry season (March-May).

Therefore, strict preventive measures should be taken in the monsoon season, when gametocytemia is at its peak, to drastically reduce the incidence of malaria.

This study also indicates a greater percentage of malaria infections in males than in females. In the Pakistani society, males are more exposed to outdoor activities, thus they are susceptible to mosquito bites. Karachi, where this study was conducted, is an industrial city and the waste water can provide breeding grounds for mosquito larvae, predisposing males to malaria infection [29-30].

Malaria causes changes in hematological parameters which are not always consistent. Anemia and thrombocytopenia are the two most common hematological observations with malaria but the WBC count may vary. Malaria transmission is greatest during the monsoon season but malaria infections in dry seasons can also be seen and it is essential to take preventive measures in order to reduce malaria incidence. One of the limitations of this study was that we only included hospitalized patients and sample size was relatively smaller.

\section{Conclusions}

In conclusion, infection with P. falciparum and P. vivax modulates significant changes in hematological parameters in populations living in malaria endemic regions. The mean platelet count was significantly lower in cases of P. vivax having gametocytes compared to P. falciparum with gametocytes. Higher parasitic index was associated with lower platelet count. The most significantly altered parameters were hemoglobin, hematocrit, WBC, and platelet count. Hemoglobin and platelets were significantly lower during the malaria season in study participants, both male and female. In the malaria season, males were more frequently affected by malaria with thrombocytopenia. Gametocyte carriage varies seasonally and may persist at low levels during the dry season.

\section{Additional Information \\ Disclosures}

Human subjects: Consent was obtained by all participants in this study. Aga Khan University Hospital issued approval 4834-Pat-ERC-17. Thank you for your response to ERC recommendations received on August 8, 2017 regarding the above mentioned study. Your response adequately answered the recommendation made by the committee. The study was accepted as exemption. You may proceed with the study. Animal subjects: All authors have confirmed that this study did not involve animal subjects or tissue. Conflicts of interest: In compliance with the ICMJE uniform disclosure form, all authors declare the following: Payment/services info: All authors have declared that no financial support was received from any organization for the submitted work. Financial relationships: All authors have declared that they have no financial relationships at present or within the previous three years with any organizations that might have an interest in the submitted work. Other relationships: All authors have declared that there are no other relationships or activities that could appear to have influenced the submitted work.

\section{References}

1. World malaria report 2015. (2016). Accessed: March 12, 2019: https://apps.who.int/iris/bitstream/handle/10665/200018/9789241565158_eng.pdf?sequence=1.

2. McKenzie FE, Wongsrichanalai C, Magill AJ, et al.: Gametocytemia in Plasmodium vivax and Plasmodium falciparum infections. J Parasitol. 2006, 92:1281-1285. 10.1645/GE-911R.1

3. Joice R, Nilsson SK, Montgomery J, et al.: Plasmodium falciparum transmission stages accumulate in the human bone marrow. Sci Transl Med. 2014, 6:244-245. 10.1126/scitranslmed.3008882

4. Koepfli C, Robinson LJ, Rarau P, et al.: Blood-stage parasitaemia and age determine Plasmodium falciparum and P. vivax gametocytaemia in Papua New Guinea. PLoS One. 2015, 10:e0126747. 10.1371/journal.pone.0126747

5. Nacher M, Carrara VI, Ashley E, et al.: Seasonal variation in hyperparasitaemia and gametocyte carriage in patients with Plasmodium falciparum malaria on the Thai-Burmese border. Trans R Soc Trop Med Hyg. 2004, 98:322-328. 10.1016/j.trstmh.2003.10.005

6. Bousema T, Drakeley C: Epidemiology and infectivity of Plasmodium falciparum and Plasmodium vivax gametocytes in relation to malaria control and elimination. Clin Microbiol Rev. 2011, 24:377-410. 10.1128/CMR.00051-10

7. Price R, Nosten F, Simpson JA, et al.: Risk factors for gametocyte carriage in uncomplicated falciparum malaria. Am J Trop Med Hyg. 1999, 60:1019-1023. 10.4269/ajtmh.1999.60.1019

8. Akim NI, Drakeley C, Kingo T, Simon B, Senkoro K, Sauerwein RW: Dynamics of P. falciparum gametocytemia in symptomatic patients in an area of intense perennial transmission in Tanzania. Am J Trop Med Hyg. 2000, 63:199-203. 10.4269/ajtmh.2000.63.199 
9. Trager W, Gill GS: Enhanced gametocyte formation in young erythrocytes by Plasmodium falciparum in vitro. J Protozool. 1992, 39:429-432. 10.1111/j.1550-7408.1992.tb01476.x

10. Trager W, Gill GS, Lawrence C, Nagel RL: Plasmodium falciparum: enhanced gametocyte formation in vitro in reticulocyte-rich blood. Exp Parasitol. 1999, 91:115-118. 10.1006/expr.1998.4347

11. Smalley ME, Brown J: Plasmodium falciparum gametocytogenesis stimulated by lymphocytes and serum from infected Gambian children. Trans R Soc Trop Med Hyg. 1981, 75:316-317. 10.1016/00359203(81)90348-5

12. Abdel-Wahab A, Abdel-Muhsin AM, Ali E, Suleiman S, Ahmed S, Walliker D, Babiker HA: Dynamics of gametocytes among Plasmodium falciparum clones in natural infections in an area of highly seasonal transmission. J Infect Dis. 2002, 185:1838-1842. 10.1086/340638

13. Kochar DK, Das A, Kochar A, et al.: Thrombocytopenia in Plasmodium falciparum, Plasmodium vivax and mixed infection malaria: a study from Bikaner (Northwestern India). Platelets. 2010, 21:623-627. 10.3109/09537104.2010.505308

14. Nacher M, Silachamroon U, Singhasivanon P, Wilairatana P, Phumratanaprapin W, Fontanet A, Looareesuwan S: Risk factors for Plasmodium vivax gametocyte carriage in Thailand . Am J Trop Med Hyg. 2004, 71:693-695.

15. Sowunmi A, Fateye BA: Plasmodium falciparum gametocytaemia in Nigerian children: before, during and after treatment with antimalarial drugs. Trop Med Int Health. 2003, 8:783-792. 10.1046/j.13653156.2003.01093.x

16. Talman AM, Domarle O, McKenzie FE, Ariey F, Robert V: Gametocytogenesis: the puberty of Plasmodium falciparum. Malar J. 2004, 3:24. 10.1186/1475-2875-3-24

17. Kotepui M, Phunphuech B, Phiwklam N, Chupeerach C, Duangmano S: Effect of malarial infection on haematological parameters in population near Thailand-Myanmar border. Malar J. 2014, 13:218. 10.1186/1475-2875-13-218

18. Idris M, Sarwar J, Fareed J: Pattern of malarial infection diagnosed at Ayub Teaching Hospital Abbottabad . J Ayub Med Coll Abbottabad. 2007, 19:35-36.

19. Zaidi SA, Kokab F, Bukhari IA, Nasir JA: The quantitative evidence of malarial transmission and its associates in Bahawalpur, Pakistan. J Ayub Med Coll Abbottabad. 2015, 27:164-167.

20. Akhtar S, Ahmed A, Ahmad A, Ali Z, Riaz M, Ismail T: Iron status of the Pakistani population-current issues and strategies. Asia Pac J Clin Nutr. 2013, 22:340-347. 10.6133/apjcn.2013.22.3.17

21. Makani J, Cox SE, Soka D, et al.: Mortality in sickle cell anemia in Africa: a prospective cohort study in Tanzania. PLoS One. 2011, 6:e14699. 10.1371/journal.pone.0014699

22. Coronado LM, Nadovich CT, Spadafora C: Malarial hemozoin: from target to tool. Biochim Biophys Acta. 2014, 1840:2032-2041. 10.1016/j.bbagen.2014.02.009

23. Ansari S, Haji KH, Abro A, Akhund IA, Qureshi F: Thrombocytopenia in plasmodium falciparum malaria . J Ayub Med Coll Abbottabad. 2009, 21:145-147.

24. Ladhani S, Lowe B, Cole AO, Kowuondo K, Newton CR: Changes in white blood cells and platelets in children with falciparum malaria: relationship to disease outcome. Br J Haematol. 2002, 119:839-847. 10.1046/j.1365-2141.2002.03904.x

25. Kotepui M, Phunphuech B, Phiwklam N, Chupeerach C, Duangmano S: Effect of malarial infection on haematological parameters in population near Thailand-Myanmar border. Malar J. 2014, 13:218. 10.1186/1475-2875-13-218

26. Hay SI, Guerra CA, Tatem AJ, Noor AM, Snow RW: The global distribution and population at risk of malaria: past, present, and future. Lancet Infect Dis. 2004, 4:327-336. 10.1016/S1473-3099(04)01043-6

27. Bousema T, Okell L, Shekalaghe S, et al.: Revisiting the circulation time of Plasmodium falciparum gametocytes: molecular detection methods to estimate the duration of gametocyte carriage and the effect of gametocytocidal drugs. Malar J. 2010, 9:136. 10.1186/1475-2875-9-136

28. Douglas NM, Simpson JA, Phyo AP, et al.: Gametocyte dynamics and the role of drugs in reducing the transmission potential of Plasmodium vivax. J Infect Dis. 2013, 208:801-812. 10.1093/infdis/jit261

29. Zhang Q, Lai S, Zheng C, et al.: The epidemiology of Plasmodium vivax and Plasmodium falciparum malaria in China, 2004-2012: from intensified control to elimination. Malar J. 2014, 13:419. 10.1186/1475-2875-13419

30. Beg MA, Sani N, Mehraj V, et al.: Comparative features and outcomes of malaria at a tertiary care hospital in Karachi, Pakistan. Int J Infect Dis. 2008, 12:37-42. 10.1016/j.ijid.2007.04.006 